\title{
THE SOLUTION OF THE PROBLEM OF INTEGRATION IN FINITE TERMS
}

\author{
BY ROBERT H. RISCH
}

\author{
Communicated by M. H. Protter, October 22, 1969
}

Introduction. The problem of integration in finite terms asks for an algorithm for deciding whether an elementary function has an elementary indefinite integral and for finding the integral if it does. "Elementary" is used here to denote those functions built up from the rational functions using only exponentiation, logarithms, trigonometric, inverse trigonometric and algebraic operations.

This vaguely worded question has several precise, but inequivalent formulations. The writer has devised an algorithm which solves the classical problem of Liouville. A complete account is planned for a future publication. The present note is intended to indicate some of the ideas and techniques involved.

Basic notions. We will deal exclusively with differential fields $D$ of characteristic zero; a differential field being a field endowed with a unary operation ' which satisfies the sum and product rule for derivatives. $\mathfrak{D}$ has a differential subfield $K$, called the constant field of $D$. It consists of all $\alpha \in D$ such that $\alpha^{\prime}=0$.

If $\mathscr{D}$ is a differential subfield of $\mathscr{F}$, then $\mathscr{F}$ (and any $f \in \mathscr{F}$ ) is said to be elementary over $D$ iff $\mathcal{F}=D\left(\theta_{1}, \cdots, \theta_{n}\right)$ where each $\theta_{i}$ satisfies at least one of the following conditions:

(1) $\theta_{i}$ is algebraic over $D\left(\theta_{1}, \cdots, \theta_{i-1}\right)$,

(2) $\theta_{i}^{\prime} / \theta_{i}=f^{\prime}$ for some $f \in D\left(\theta_{1}, \cdots, \theta_{i-1}\right)$ (the exponential case),

(3) $f^{\prime} / f=\theta_{i}^{\prime}$ for some $f \in D\left(\theta_{1}, \cdots, \theta_{i-1}\right)$ (the logarithmic case).

If in (2) or (3) $\theta_{i}$ is transcendental over $D\left(\theta_{1}, \cdots, \theta_{i-1}\right)$ and the constant field of $D\left(\theta_{1}, \cdots, \theta_{i-1}\right)$ is the same as that of $D\left(\theta_{1}, \cdots, \theta_{i}\right)$, then $\theta_{i}$ is a monomial over $D\left(\theta_{1}, \cdots, \theta_{i-1}\right)$. If each $\theta_{i}$ is either algebraic or a monomial over $D\left(\theta_{1}, \cdots, \theta_{i-1}\right)$ then $\mathcal{F}$ is regular elementary over D.

(1), (2) and (3) are all the operations needed since we get the trigonometric and inverse trigonometric operations by adjoining $\sqrt{ }(-1)$ to $K$.

The following basic result gives us the form assumed by elementary

AMS Subject Classifications. Primary 1280, 3402; Secondary 1451.

Key Words and Phrases. Elementary function, integration in finite terms, algorithm, differential field, Jacobian variety, bounding torsion. 
functions that have elementary indefinite integrals. For a proof see [8, p. 171].

LIOUVILLE'S THEOREM. Let $\mathfrak{F}$ be a differential field with an algebraically closed constant field $K$. Let $f \in \mathcal{F}$ and $g$ be elementary over $\mathcal{F}$ with $g^{\prime}=f$. Then there are $v_{0}, v_{1}, \cdots, v_{k}$ in $\mathcal{F}$ and $c_{1}, \cdots, c_{k}$ in $K$ such that $f=v_{0}^{\prime}+\sum c_{i} v_{i}^{\prime} / v_{i}$.

Brief description of the algorithm. For a differential field of the type $\mathcal{F}=K\left(z, \theta_{1}, \cdots, \theta_{m}\right)$ where $K$ is the constant field of $\mathscr{F}, K$ being algebraically closed and of finite transcendence degree over $Q, z^{\prime}=1$ with $F$ regular elementary over $K(z)$, the integration algorithm gives a method for determining whether a given $f \in \mathcal{F}$ can be represented in the form $v_{0}^{\prime}+\sum c_{i} v_{i}^{\prime} / v_{i}$, the $v_{i}^{\prime}$ 's being in $\mathcal{F}$ and the $c_{i}^{\prime}$ 's in $K$.

The algorithm proceeds by induction on the number of monomials used in constructing a tower from $K(z)$ to $F$. Let $D$ be elementary over $K(z)$ where $m-1$ monomials are used in a tower from $K(z)$ to D. The induction hypothesis assumes that we can decide the elementary integrability of elements of $D$ and also tell if a first order linear differential equation with coefficients in $\mathfrak{D}$, having certain constant parameters, has a solution in $D$.

Now let $\theta$ be a monomial over $\mathscr{D}$, $w$ be algebraic over $D(\theta)$ and $f \in \mathcal{F}$ where $\mathcal{F}=\mathbb{D}(\theta, w)$. We examine the equation $f=v_{0}^{\prime}+\sum c_{2} v_{i}^{\prime} / v_{i}$ at a finite set (determined by $f$ ) of $D$-places of $\mathcal{F}$. Using the induction hypothesis, certain necessary conditions are obtained for the $v$ 's occurring in a suitable Liouville representation of $\mathscr{F}$; namely, a principal part system for $v_{0}$ and divisors $\delta_{i}, i=1, \cdots, k$ such that $\left(v_{i}\right)$ is a power of $\delta_{i}$. The $v^{\prime}$ s, if they exist, can be determined up to additive or multiplicative elements of $D$. Then the problem is reduced to studying an equation $f_{1}=d_{0}^{\prime}+\sum c_{i} d_{i}^{\prime} / d_{i}$ where $f_{1}$ and the $d^{\prime}$ 's are in $D$.

The case $\mathcal{F}=K\left(z, \theta_{1}, \cdots, \theta_{m}\right)$ where each $\theta_{i}$ is a monomial over $K\left(z, \theta_{1}, \cdots, \theta_{i-1}\right)$ was treated in detail in [8]. All the algebraic function fields involved are rational and the above method reduces to a generalization of the usual partial fraction method used for integrating elements of $K(z)$.

In all the foregoing it is assumed that one has an explicit transcendance basis for the field $K$ so problems dealing with transcendental numbers are avoided. For example, since there is no known method that tells for an arbitrary $r \in Q$ whether $e^{e}=r$, there is no known general method for telling if an integral of the form $\int\left(e^{b}-r\right) e^{z^{2}}$ is elementary.

The restriction to regular elementary extensions of $K(z)$ avoids adjoining new transcendental constants and also prevents certain 
insurmountable difficulties associated with the multiple valued character of the logarithm function. (Cf. [8, Proposition 2.2].)

Integration and torsion on the Jacobian variety. The most surprising aspect of the integration problem is the fact (implicit in the above) that it reduces to the problem of bounding torsion on the Jacobian variety of an algebraic curve. We will explain this more fully for the case of Abelian integrals, i.e., integration of elements of $\mathfrak{F}=K(z, w)$, where $f(z, w)=0, f$ being a polynomial with coefficients in $K$.

The following can be derived from Liouville's theorem:

Let $\omega$ be a differential of $\mathcal{F}$. Let $\boldsymbol{r}_{1}, \cdots, \boldsymbol{r}_{\boldsymbol{k}}$ be a basis for the $\boldsymbol{Z}$ module generated by the residues of $\omega$. Thus at each $K$-place $p, \operatorname{Res}_{p}(\omega)=$ $a_{1 p} r_{1}+\cdots+a_{k p} r_{k}, a_{i p} \in Z$. Let the divisor $\delta_{i}$ be given by $\prod_{p} p^{a_{i p}}$. Suppose $\int \omega$ is elementary. Then there are $v_{0}, \cdots, v_{k}$ in $\mathcal{F}$ and integers $j_{1}$, $\cdots, j_{k}$ such that $\delta^{j_{i}}=\left(v_{i}\right)$ and $\omega=d v_{0}+\sum_{i=1}^{k}\left(r_{i} / j_{i}\right)\left(d v_{i} / v_{i}\right)$.

All of the divisors $\delta_{i}$ and also the coefficients of $f$ will be rational over a field $k \subset K, k$ being finitely generated over $Q$. Thus we can work with the field $k(z, w)$. If $B$ is a bound for the order of the torsion subgroup of the Jacobian variety of $k(z, w)$, then it can be assumed that each $j_{i} \leqq B$. Then one must test to see if any of $\delta_{i}, \delta_{i}^{2}, \cdots, \delta_{i}^{B}$ is principal. Either the classic arithmetic or geometric theories of algebraic functions give constructive criteria for the latter question. E.g. [1, Chapter III].

In the above special case of Abelian integrals, the fact that integration reduces to divisor testing was first explicitly (albeit, somewhat obliquely) stated by Goursat in 1894 [5, p. 516]. At that time the problem was considered exceedingly difficult or even undecidable (before Gödel!). See the remarks of Halphen [6, last page], Goursat [5, p. 516], and Hardy [7, pp. 8-11, 47-48, 52]. The only criteria they considered was the highly nonconstructive one given by Abel's theorem.

A bound for the torsion. It has been conjectured that there is a universal bound, depending only on the genus and the ground field for torsion on the Jacobian variety of an algebraic curve defined over a finitely generated field $k$. (See $[2$, p. 264] for some discussion of the elliptic curve case.) When integrating a given elementary function, one needs only to be able to find the bound for an explicitly given curve. This can be done using things now in the repertory of arithmetical algebraic geometry. One method is outlined below.

Let $C$ be a nonsingular, projective model of an absolutely irreducible algebraic curve defined over $k$. Since $k$ is finitely generated over the rationals, it can be embedded in a finite algebraic extension of the $p$-adic numbers. $C$ can then be reduced $\bmod \pi$ where $\pi$ is a valuation 
of $k$, extending the $p$-adic valuation of $Q$. We wish this reduction to be "good." That is, a finite set of forms defining a generic point for $C$ should reduce $\bmod \pi$ to a set of forms that also give a nonsingular, absolutely irreducible curve $C^{1}$. A sufficient condition for this is that $\pi$ be constructed so that $\operatorname{ord}_{\pi}\left(\beta_{i}\right) \geqq 0$ for a certain set of elements $\beta_{1} \cdots \beta_{b}$ of $k$ with $\operatorname{ord}_{\pi}\left(\beta_{1}\right)=0$.

In a good reduction, the group of divisor classes on $C$, of finite order $l$, where $(l, p)=1, p$ being the characteristic of the residue class field, is injected into the corresponding group on $C^{1}$. This last assertion follows from Shimura's result [9, Proposition 16, p. 98], if we note that the good reduction can be extended to one from $g(C)$ to $g\left(C^{1}\right)$, these being the corresponding Chow models of the Jacobian $[3, \mathrm{p}$. $651]$.

Thus we will have a bound for the torsion if we can find the class numbers of two good reductions $C^{1}$ and $C^{2}$ which are defined over residue class fields of different characteristics. But this can be obtained using the rationality formula for the zeta function $[4$, Chapter $\mathrm{V}, \S \S 5.1,5.2]$.

I would like to express my indebtedness to Professor James Ax for his advice and suggestions during the writing of this note. I would also like to thank Professor Goro Shimura for information concerning good reduction.

\section{REFERENCES}

1. G. A. Bliss, Algebraic functions, Dover, New York, 1966. MR 34 \#2866.

2. J. W. S. Cassels, Diophantine equations with special reference to elliptic curves, J. London Math. Soc. 41 (1966), 193-291. MR 33 \#7299.

3. W.-L. Chow and S. Lang, On the birational equivalence of curves under specialization, Amer. J. Math. 79 (1957), 649-652. MR 19, 767.

4. M. Eichler, Introduction to the theory of algebraic numbers and functions, Birkhaüser, Basel, 1963; English transl., Pure and Appl. Math., vol. 23, Academic Press, New York, 1966. MR 29 \#5821; MR 35 \#160.

5. E. Goursat, Sur les intégrales abeliennes qui s'experiment par logarithms, C.R. Acad. Sci. Paris 118 (1894), 515-517.

6. G. H. Halphen, Traité des fonctiones elliptiques et de leurs applications. Deuxiéme Partie, Gauthier-Villars, Paris, 1888.

7. G. H. Hardy, The integration of functions of a single variable, 2nd ed., Cambridge Univ, Press, New York, 1916.

8. R. H. Risch, The problem of integration in finite terms, Trans. Amer. Math. Soc. 139 (1969), 167-189. MR 38 \#5759.

9. G. Shimura and Y. Taniyama, Complex multiplication of abelian varieties and its applications to number theory, Publ. Math. Soc. Japan, 6, The Mathematical Society of Japan, Tokyo, 1961. MR 23 \#A2419. 10598

ibm Thomas J. Watson Research Center, Yorktown Heights, New York 